\section{Alcohol consumption and smoking dose-dependently and synergistically worsen local pancreas damage}

Chronic pancreatitis (CP) is characterised by irreversible damage to the pancreas causing endocrine and exocrine dysfunction which results in decreased quality of life and reduced life expectancy. ${ }^{1}$

Adam et al recently published an interesting study on a possible diagnostic tool for CP based on metabolomic profiles of patients and controls. ${ }^{2}$

Our previous international cohort analysis showed that the proportion of patients developing CP is exponentially and directly associated with the number of acute pancreatitis (AP) episodes, thus strengthening the focus on the challenging task of diagnosing CP early. ${ }^{34}$ However, in addition to diagnosing early, we should also focus on preventive interventions, before the damage becomes irreversible.

Alcohol is the main aetiological factor for CP and both alcohol consumption and smoking increase the risk for recurrence of $\mathrm{AP}$ and the development of CP. According to Ahmed Ali et al, in a follow-up study of 669 AP patients, smoking represented the dominant risk factor for recurrent AP (RAP) and a combination of alcohol consumption and smoking was the main risk factor for the progression to $\mathrm{CP}^{5}$ Therefore, cessation programmes and patient education are extremely important means to intervening and lowering the recurrence of AP and the progression to $\mathrm{CP}^{67}$ However, total cessation and abstinence often seems impossible for patients and they do not even try. Is it also possible to reduce recurrence and progression by decreasing the amount consumed?

Basic research evidence clearly suggests that alcohol and smoking amplify each other's harmful effects. ${ }^{89}$ However, large cohorts are lacking to determine whether smoking and alcohol consumption dosedependently, mutually exacerbate the damage to the pancreas caused by each.

We have used the international cohort in the Acute Pancreatitis Registry initiated by the Hungarian Pancreatic Study Group. Data were collected from 13 countries and 30 medical centres, with 2441 cases included in the analysis. Further characteristics of the cohort and information on methods are available in online supplemental file 1.

The patient population was divided into groups according to current amounts of smoking and alcohol consumption. We found that both smoking and alcohol
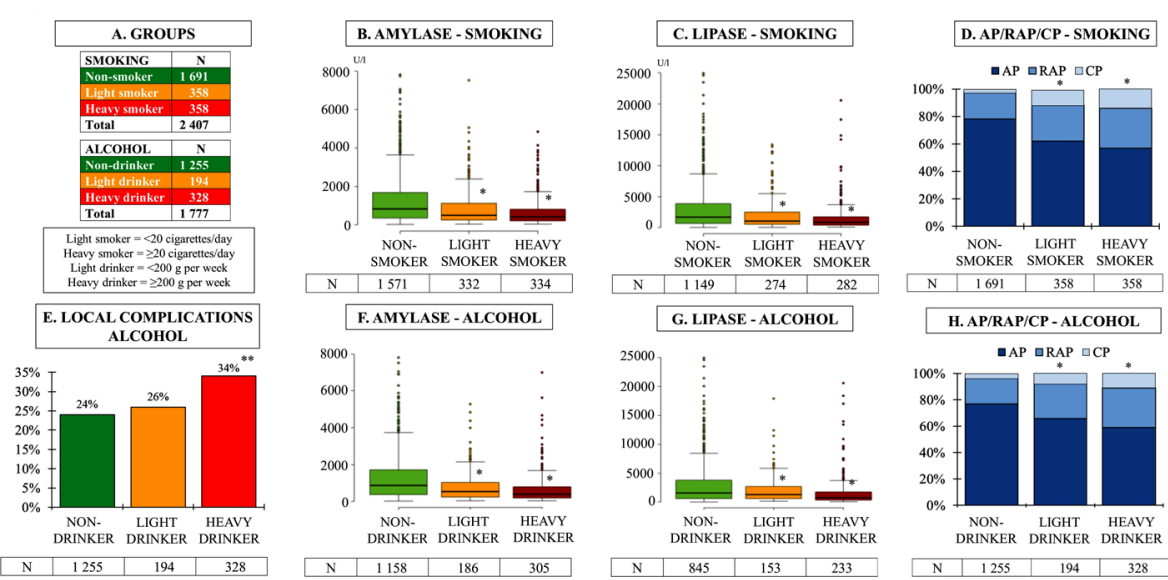

Figure 1 Dose dependency in alcohol consumption and smoking in AP. (A) Light and heavy alcohol consumption and smoking groups and definitions. (B, C) Amylase and lipase levels on admission. (D) Prevalence of RAP and CP (\%). (E) Proportion of local complications (\%). $(F, G)$ Amylase and lipase levels on admission. $(H)$ Prevalence of RAP and $C P(\%)$. ${ }^{*} P<0.001$, ${ }^{* *} \mathrm{p}=0.003$. Pearson's $\chi^{2}$ test and the Kruskal-Wallis rank sum tests were used. AP, acute pancreatitis; $C P$, chronic pancreatitis; RAP, recurrent $A P$.

consumption are dose-dependently associated with amylase and lipase levels and with the prevalence of RAP and CP among AP patients. Alcohol consumption was also linked to a higher rate of local complications (figure 1).

Second, we examined the possible synergistic effect of these two risk factors. We arranged the cohort population into four groups based on current smoking and alcohol consumption status (figure 2). Smoking and drinking together are associated with the male sex and linked to the first AP episode 15 years earlier than non-smoking and nondrinking are. Analysing on-admission and
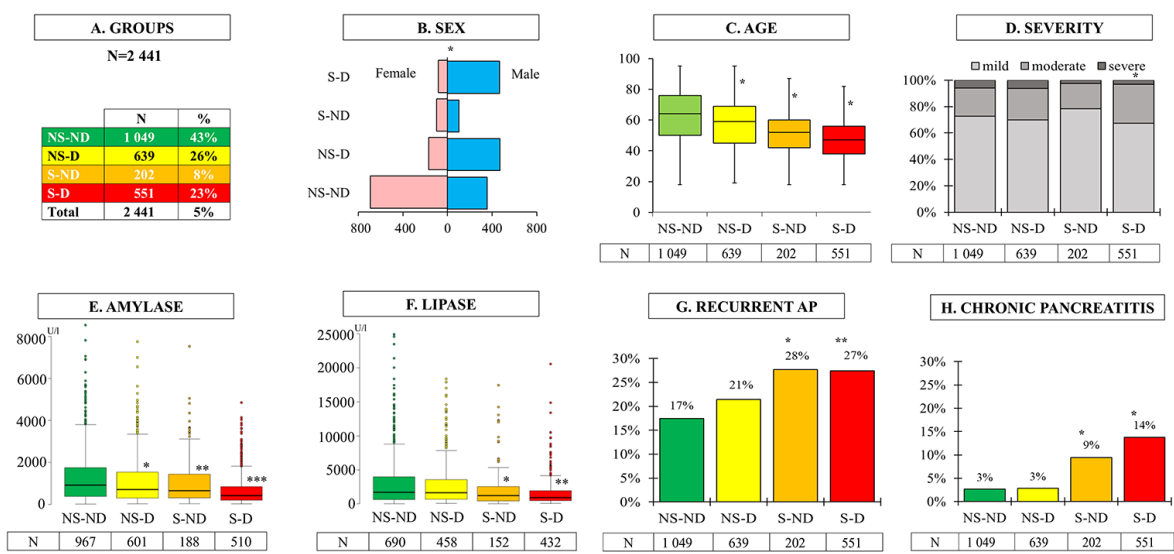

Figure 2 Combined alcohol consumption and smoking groups in AP. (A) Groups. (B) Sex. ${ }^{*} \mathrm{P}<0.001$ in all comparisons. (C) Age. ${ }^{*} \mathrm{P}<0.001$ or $\mathrm{p}=0.001$ in all comparisons. (D) Severity. Moderately severe ${ }^{*} p=0.003$ vs NS-ND. $P=0.022$ vs $S-N D$. (E) On admission amylase level. ${ }^{*} \mathrm{P}<0.001$ vs NS-ND. ${ }^{*} \mathrm{P}<0.003$ vs NS-ND. ${ }^{* * *} \mathrm{p}<0.001$ vs NS-ND, NS-D and S-ND. (F) Onadmission lipase level. ${ }^{*} \mathrm{P}<0.003$ vs NS-ND, $\mathrm{p}<0.006$ vs NS-D. ${ }^{*} \mathrm{P}<0.001$ vs NS-ND and NS-D.

(G) Prevalence of recurrent AP. * $P=0.003$ vs NS-ND. ${ }^{*} p=0.04$ vs NS-D. $p<0.001$ vs NS-ND. (H) Prevalence of chronic pancreatitis. ${ }^{*} P<0.001$ vs NS-ND, NS-D. Pearson's $\chi^{2}$ test and the Kruskal-Wallis rank sum tests were used. AP, acute pancreatitis; NS-D: non-smoking-drinking; NS-ND: non-smoking-non-drinking; S-D: smoking-drinking; S-ND: smoking-non-drinking. 
Our analysis confirms in a clinical setting that both smoking and alcohol are dosedependently associated with pancreatic tissue damage and the prevalence of RAP and CP. Moreover, they mutually exacerbate each other's harmful effect. In addition to the development of prognostic and therapeutic measures, further clinical trials on cessation programmes and patient education are needed. Communication to all stakeholders of the importance of at least quitting smoking or cutting the amount of smoking and alcohol consumption is crucial.

Andrea Szentesi, ${ }^{1,2}$ Nelli Farkas, ${ }^{1,3}$ Zoltán Sipos, ${ }^{1}$ Péter Mátrai, ${ }^{1}$ Áron Vincze, ${ }^{4}$ Ferenc Izbéki, ${ }^{5}$ Andrea Párniczky, ${ }^{1,6}$ Péter Hegyi $\odot{ }^{1,7,8}$ Hungarian Pancreatic Study Group

${ }^{1}$ Institute for Translational Medicine, Szentágothai Research Centre, Medical School, University of Pécs, Pécs, Hungary

${ }^{2}$ Centre for Translational Medicine, Department of Medicine, University of Szeged, Szeged, Hungary ${ }^{3}$ Institute of Bioanalysis, Medical School, University of Pécs, Pécs, Hungary

${ }^{4}$ Division of Gastroenterology, First Department of Medicine, Medical School, University of Pécs, Pécs, Hungary

${ }^{5}$ Szent György University Teaching Hospital of Fejér County, Székesfehérvár, Hungary

${ }^{6}$ Heim Pál National Pediatric Institute, Budapest, Hungary

${ }^{7}$ Division of Pancreatic Diseases, Heart and Vascular Center, Semmelweis University, Budapest, Hungary ${ }^{8}$ Centre for Translational Medicine, Semmelweis University, Budapest, Hungary

Correspondence to Professor Péter Hegyi, Division of Pancreatic Diseases, Heart and Vascular Center, Semmelweis University, Budapest, Hungary; hegyi2009@gmail.com

Acknowledgements We wish to thank all the contributing members of the Hungarian Pancreatic Study Group (HPSG) and the Centre of Excellence for Pancreatic Diseases (CEPD). The abstract for the analysis was published at conferences held by the European Pancreatic Club, United European Gastroenterology and the American Pancreatic Association.

Collaborators Hungarian Pancreatic Study Group: Bálint Erőss, Péter Jenő Hegyi, Szilárd Váncsa, Rita Nagy, Katalin Márta, Klementina Ocskay, Márk Félix Juhász, Marcell Imrei, Mária Földi, Szabolcs Kiss, Balázs Csaba Németh, Tamás Takács, László Czakó, Szilárd Gódi, Judit Bajor, Patrícia Sarlós, László Gajdán, Mária Papp, József Hamvas, Márta Varga, Melania Macarie, Imola Török, János Novák, Artautas Mickevicius, Elena Ramirez Maldonado, Shamil Galeev, Ville Sallinen, Barnabás Bod, Ali Tüzün Ince, Tamás Nagy, Nándor Faluhelyi, Noémi Gede, Stefania Bunduc, Tamás Hussein, Mónika Lipp, Anna Németh, Orsolya Urbán, Dorottya Tarján, Simon Tóth, Dániel Pécsi, Péter Varjú, Noémi Zádori.
Contributors PH conceptualised the study. ÁV, FI, AS and AP contributed to the data collection and quality assurance. AS, ZS, PM and NF extracted and analysed the data. PH, AS, AP and NF interpreted the data. AS and $\mathrm{PH}$ wrote the manuscript. All the authors reviewed and contributed to the manuscript before finalisation and submission. Hungarian Pancreatic Study Group (full names are available in the Contributors section and affiliations are detailed in online supplemental file 1: BE, PJH, SV, RN, KM, KO, FJ, MF, SK, BN, TT, LC, SG, JB, PS, LG, MP, JH, MV, MM, IT, JN, AM, ERM, SG, VS, BB, ATI contributed to the data collection. TN, NF contributed to the interdisciplinary evaluation of the cases. NG conducted preliminary analyses. BE, PJH, SV, RN, KM, KO, FJ, MI, MF, SK, NG, SB, TH, ML, AN, OU, DT, ST, DP, PV and NZ ensured professional data quality control.

Funding The research was supported by project grant $\mathrm{K} 131996$ to PH and by funding from the University of Pécs Medical School Research Fund (300909) to AS.

Disclaimer The funders had no effect on the concept, data collection and analysis, and writing of the manuscript.

Competing interests None declared.

Patient consent for publication Not applicable.

Ethics approval The study was approved by the Scientific and Research Ethics Committee of the Medical Research Council and the National Public Health Centre under the following ID numbers: 222541/2012/EKU and 17787-8/2020/EÜIG.

Provenance and peer review Not commissioned; internally peer reviewed.

Data availability statement Original raw data are available from the corresponding author on reasonable request.

Supplemental material This content has been supplied by the author(s). It has not been vetted by BMJ Publishing Group Limited (BMJ) and may not have been peer-reviewed. Any opinions or recommendations discussed are solely those of the author(s) and are not endorsed by BMJ. BMJ disclaims all liability and responsibility arising from any reliance placed on the content. Where the content includes any translated material, BMJ does not warrant the accuracy and reliability of the translations (including but not limited to local regulations, clinical guidelines, terminology, drug names and drug dosages), and is not responsible for any error and/or omissions arising from translation and adaptation or otherwise.

\section{(2) OPEN ACCESS}

Open access This is an open access article distributed in accordance with the Creative Commons Attribution Non Commercial (CC BY-NC 4.0) license, which permits others to distribute, remix, adapt, build upon this work non-commercially, and license their derivative works on different terms, provided the original work is properly cited, appropriate credit is given, any changes made indicated, and the use is non-commercial. See: http:// creativecommons.org/licenses/by-nc/4.0/.

(C) Author(s) (or their employer(s)) 2022. Re-use permitted under CC BY-NC. No commercial re-use. See rights and permissions. Published by BMJ.

- Additional supplemental material is published online only. To view, please visit the journal online (http://dx.doi.org/10.1136/gutjnl-2021-326853).

\section{Check for updates}

To cite Szentesi A, Farkas N, Sipos Z, et al. Gut Epub ahead of print: [please include Day Month Year]. doi:10.1136/gutjnl-2021-326853

Received 26 December 2021

Accepted 4 January 2022

Gut 2022;0:1-2. doi:10.1136/gutjnl-2021-326853

ORCID iD

Péter Hegyi http://orcid.org/0000-0003-0399-7259

\section{REFERENCES}

1 Beyer G, Habtezion A, Werner J, et al. Chronic pancreatitis. The Lancet 2020;396:499-512.

2 Adam MG, Beyer G, Christiansen N, et al. Identification and validation of a multivariable prediction model based on blood plasma and serum metabolomics for the distinction of chronic pancreatitis subjects from non-pancreas disease control subjects. Gut 2021;70:2150-8

3 Erőss B, Szentesi A, Hegyi P. Metabolic signature might be an option to identify patients with early CP. Gut 2021;70:2023-4.

4 Hegyi PJ, Soós A, Tóth E, et al. Evidence for diagnosis of early chronic pancreatitis after three episodes of acute pancreatitis: a cross-sectional multicentre international study with experimental animal model. Sci Rep 2021;11:1-14

5 Ahmed Ali U, Issa Y, Hagenaars JC, et al. Risk of recurrent pancreatitis and progression to chronic pancreatitis after a first episode of acute pancreatitis. Clin Gastroenterol Hepatol 2016;14:738-46.

6 Nordback I, Pelli H, Lappalainen-Lehto R, et al. The recurrence of acute alcohol-associated pancreatitis can be reduced: a randomized controlled trial. Gastroenterology 2009;136:848-55.

7 Ocskay K, Juhász MF, Farkas N, et al. Recurrent acute pancreatitis prevention by the elimination of alcohol and cigarette smoking (REAPPEAR): protocol of a randomised controlled trial and a cohort study. BMJ Open 2022;12:e050821.

8 Lugea A, Gerloff A, Su H-Y, et al. The combination of alcohol and cigarette smoke induces endoplasmic reticulum stress and cell death in pancreatic acinar cells. Gastroenterology 2017;153:1674-86.

9 Sahin-Tóth M, Hegyi P. Smoking and drinking synergize in pancreatitis: multiple hits on multiple targets. Gastroenterology 2017;153:1479-81. 